\title{
Measuring Sleep, Wakefulness, and \\ Circadian Functions in Neurologic Disorders
}

Markus H. Schmidt, MD, PhD ${ }^{a, b}, *, 1$, Martijn P.J. Dekkers, MD, PhD ${ }^{a, 1}$,

Sébastien Baillieul, MD, PhD ${ }^{a, c, 1}$, Jasmine Jendoubi, $\mathrm{MSc}^{\mathrm{a}, 1}$,

Marie-Angela Wulf, MD, PhD ${ }^{a, 1}$, Elena Wenz, MD ${ }^{a, 1}$, Livia Fregolente, MD ${ }^{a, 1}$,

Albrecht Vorster, $\mathrm{PhD}^{\mathrm{a}, 1}$, Oriella Gnarra, MSc ${ }^{\mathrm{a}, \mathrm{d}, 1}$,

Claudio L.A. Bassetti, MD ${ }^{a, e, 1}$

\section{KEYWORDS}

- Sleep $\bullet$ Wakefulness $\bullet$ Circadian $\bullet$ Stroke $\bullet$ Neurodegenerative disorders $\bullet$ Neuroimmunology

- Polysomnography • Sleep architecture

\section{KEY POINTS}

- Neurologic disorders impact the ability of the brain to generate sleep, wake, and circadian functions.

- Preexisting or de novo sleep-wake-circadian pathologies are generally underdiagnosed in neurologic patients despite their major impact on onset, evolution, and outcome of neurologic disorders.

- Neurologic disorders are frequently accompanied by sleep-wake EEG changes. Extensive brain damage can lead to the absence of measurable differentiation between sleep and wakefulness (status dissociatus).

- New technologies will facilitate early detection and (long-term) monitoring of neurologic patients and the optimization of their clinical management.

\section{INTRODUCTION}

The brain is the organ from which sleep, wakefulness, and circadian functions are generated and ultimately measured. The highly structured and timed transitions between wakefulness, nonrapid eye movement (NREM) sleep, and REM sleep requires an integration of neural networks across many brain structures, including the brainstem, subcortical regions such as the thalamus and hypothalamus, and basal forebrain.
Central nervous system (CNS) lesions underlying neurologic disorders can lead to primary sleep-wake and circadian disorders (SWCD) through lesioning of specific cell types or structures generating or regulating sleep, wake, and circadian functions or through nonspecific lesioning of diffuse neural networks. In addition, SWCD can arise secondarily from complications of CNS lesions such as spasticity, pain, and depression. In many cases SWCD may worsen over time, as in the case of progressive neurologic diseases.

\footnotetext{
a Department of Neurology, Bern University Hospital (Inselspital) and University Bern, Switzerland; ${ }^{b}$ Ohio Sleep Medicine Institute, 4975 Bradenton Avenue, Dublin, OH 43017, USA; ' Univ. Grenoble Alpes, Inserm, U1300, CHU Grenoble Alpes, Service Universitaire de Pneumologie Physiologie, Grenoble 38000, France; d Sensory-Motor System Lab, IRIS, ETH Zurich, Switzerland; ' Department of Neurology, University of Sechenow, Moscow, Russia

1 Present address: Schlaf-Wach-Epilepsie Zentrum (SWEZ), Universitätsklinik für Neurologie, Bern University Hospital, Inselspital, Freiburgstrasse 4, 3010 Bern, Switzerland.

* Corresponding author. Inselspital, Universitätsklinik für Neurologie CH-3010 Bern

E-mail address: markus.schmidt@insel.ch
} 
Finally, SWCD may also represent the first or main manifestation of an underlying neurologic disorder such as dream enactment behavior in Parkinson disease (PD), excessive daytime sleepiness (EDS) in hypothalamic disorders, or insomnia in Alzheimer disease (AD).

Lesions of the various structures that regulate sleep, wake, and circadian functions will lead to specific changes. For example, lesions of the brainstem may affect aspects of REM sleep generation or expression, thalamic lesions can lead to a reduction of spindling, and damage to the suprachiasmatic nucleus may disrupt circadian rhythmicity. These various pathologic manifestations can be measured through subjective assessments, including questionnaires, and objective tools using actigraphy, polysomnography (PSG), or daytime vigilance testing (Fig. 1). The diversity of SWCD in neurologic patients, which may reflect brain damage or other factors such as comorbidities or medication, and their measurement in clinical practice are the subjects of this article.

\section{STROKE}

Stroke is one of the leading causes of death and disability worldwide ${ }^{1}$ and is often associated with significant changes in sleep-wake electroencephalographic (EEG) architecture and circadian expression. ${ }^{2,3}$ In addition, SWCD are also increasingly recognized as stroke risk factors and modulators of stroke outcome. ${ }^{2,4,5}$ Therefore, SWCD diagnosis and management should be considered in stroke care pathways.

\section{Subjective Assessments}

The simplest way to assess subjective SWCD in patients with stroke is by using structured interview questions. A detailed sleep history addressing sleep habits before and following stroke, including estimated sleep needs (hours per day), can be easily gathered from patients or their relatives, even at hospital admission. ${ }^{6,7}$ However, limited studies have assessed subjective sleep duration prestroke and poststroke and heralded conflicting results. A systematic study from our group conducted in 438 patients suggested an increased sleep duration following ischemic stroke with a gradual, but incomplete, return to baseline at 12 months. $^{8}$ An increase in sleep duration or need is particularly often found in patients with (bilateral) lesions of the paramedian thalamus. $^{6,9}$

Symptoms such as fatigue, insomnia, and EDS are common after stroke. ${ }^{10,11}$ Restless legs syndrome (RLS), in contrast, does not seem to be more prevalent after stroke than in the general population. ${ }^{12}$ In a systematic study from our center over a follow-up period of 2 years, fatigue was found in up to $28 \%$, insomnia in $28 \%$, EDS in $14 \%$, and RLS in $9 \%$ of patients with stroke (manuscript in preparation).

Sleep questionnaires such as the Epworth Sleepiness Scale, Fatigue Severity Scale, Insomnia

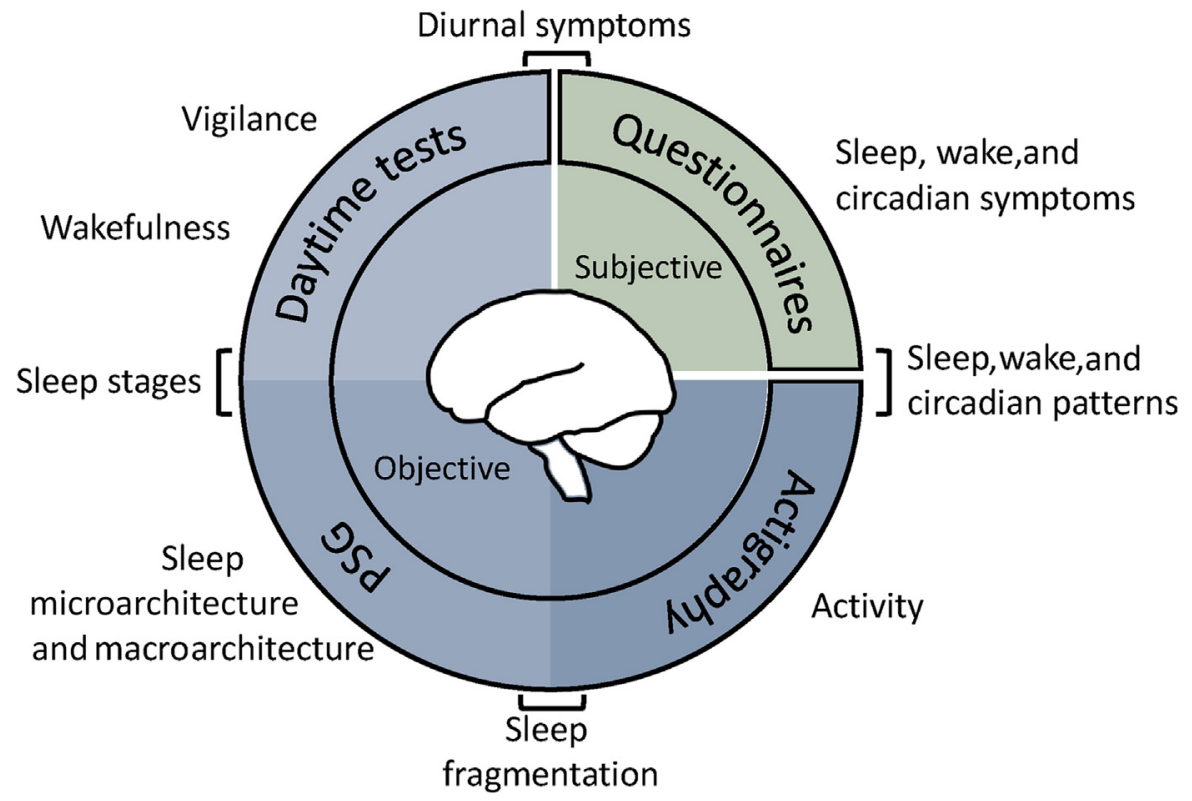

Fig. 1. Measuring sleep-wake and circadian disorders using subjective and objective assessments in neurologic disease. 
Severity Index, and the Pittsburgh Sleep Quality Index Questionnaire ${ }^{9,13,14}$ can also be used to help investigate specific symptoms of SWCD. It is important to note, however, that validation of these commonly used questionnaires as part of a test battery in patients with stroke is lacking. Moreover, the use of questionnaires for sleep-disordered breathing (SDB) has shown limitations in patients with stroke with their high sensitivity but a rather low specificity. ${ }^{15}$ Taken together, although the use of questionnaires validated for the general population may provide potential screening tools for SWCD evaluation, objective assessments remain frequently necessary in a stroke population in which SWCD are highly prevalent. ${ }^{16}$

\section{Objective Assessments}

Circadian functions can be assessed using actigraphy. A disruption of circadian rhythmicity, as measured by core body temperature and actigraphy acutely after stroke, correlated strongly with functional outcome 3 months after stroke onset. ${ }^{17}$ In addition to estimating macroparameters of sleep-wake rhythms, wearable devices may offer the possibility for long-term home monitoring, allowing the investigation of sleep-wake behavior evolution after stroke, as well as measuring the effect or efficacy of therapeutic interventions (see section 6.2). ${ }^{18}$

In-laboratory PSG demonstrates that sleep macroarchitectural changes following stroke potentially affect all sleep and EEG variables. ${ }^{2,11}$ For example, supratentorial stroke may be followed by a reduction in REM sleep in a stroke severity-dependent manner and may persist for months. ${ }^{19,20}$ Brainstem stroke lesions affecting the ventrolateral and tegmental areas in the pons can also reduce REM sleep or lead to REM sleep behavior disorder (see later), especially in the case of bilateral lesions. ${ }^{21}$ Noteworthy, stroke in these regions may lead to a reduction in NREM sleep even in the absence of subjective sleep complaints. ${ }^{21}$

Thalamic strokes predominantly reduce sleep spindles and deeper NREM sleep stages. ${ }^{6,9,22,23}$ Indeed, a reduction in sleep spindles after thalamic and supratentorial strokes ${ }^{24,25}$ may occur as a function of lesion size. ${ }^{19}$ Moreover, spindle power reduction in the peri-infarct area acutely after stroke is accompanied by a temporarily increased spindle power over the contralesional hemisphere.

In acute and chronic stroke, local slow-wave activity (SWA) is increased over the infarct area and decreased over the peri-infarct area during sleep ${ }^{25}$; this is of interest for different reasons. In a recent study we observed an association between a reduction of the slow-wave slope in patients with thalamic stroke and daytime sleepiness. ${ }^{26}$ In addition, SWA is increasingly linked with sleep-related learning and memory, ${ }^{27,28}$ processes that could play a role in neuroplasticity after stroke. ${ }^{5}$ In support of this hypothesis, optogenetically induced slow waves delivered during sleep were reported to improve functional outcomes in a mouse model of stroke. ${ }^{29}$ Together, these data provide a conceptual framework for therapeutic noninvasive neuromodulation approaches targeting (slow wave) sleep after stroke (and overall brain injury).

Only a few studies objectively investigated the impact of stroke on daytime vigilance using the multiple sleep latency test (MSLT) and the maintenance of wakefulness test (MWT). Sleep latencies were reduced in subcortical stroke, ${ }^{30}$ whereas the latencies did not necessarily correlate with subjective measures of EDS in patients with bilateral thalamic lesions. ${ }^{22}$ These data reveal potential discrepancies between subjective questionnaires and objective monitoring that may reflect a discordance between perceived alertness and actual waking ability. Such discrepancies can also be observed in the general population ${ }^{31}$ and reflect the need for objective assessments of daytime alertness. However, objective testing can be challenging because large supratentorial lesions may also limit the expression of EEG correlates of sleep-wake behaviors (see section Neurodegenerative Disorders).

\section{NEURODEGENERATIVE DISORDERS}

Sleep, wake, and circadian disturbances are a common finding in neurodegenerative diseases. ${ }^{32}$ As neurodegenerative diseases often affect the neuronal networks underlying the generation of sleep, wakefulness, and circadian timing, standard methods to measure and score sleep and wakefulness are potentially challenging, ${ }^{33}$ and this was dramatically documented by Landolt and colleagues $^{33}$ in study of patients with sleep-wake disturbances in the course of Creutzfeldt-Jakob disease. Similarly, Santamaria and colleagues ${ }^{34}$ emphasized the importance of audiovideographic recordings, including calibration of measured variables with clearly awake periods with major sleep and wake bouts in patients with brain damage. ${ }^{34}$

\section{Subjective Assessments}

Up to $60 \%$ of patients with $A D$ and $90 \%$ of patients with PD suffer from insomnia. ${ }^{35,36}$ EDS is also frequent and may affect more than $40 \%$ to $50 \%$ of both patients with $A D$ and those with PD. ${ }^{36,37}$ REM sleep behavior disorder (RBD) is frequent in 
$\mathrm{PD}^{38}$ but rare in $\mathrm{AD} .^{39}$ In RBD, the complex neural networks controlling muscle atonia or paralysis during REM sleep are disrupted, leading to motor activation during this stage of sleep. If RBD occurs in the context of a neurodegenerative disorder, such as PD, or is triggered by medication, it is classified as secondary RBD. However, if no underlying cause can be identified, it is known as idiopathic RBD (iRBD), which can appear in the prodromal phase of $\alpha$-synucleinopathies. Finally, RLS is probably more prevalent in patients with PD compared with healthy individuals, ${ }^{40}$ and, in patients with $A D$, RLS may manifest as nighttime agitation. $^{32}$

Several questionnaires for RBD have been developed, including the RBD screening questionnaire (RBDSQ), which is the most commonly used. ${ }^{38}$ A meta-analysis of the RBDSQ demonstrated a sensitivity and specificity of $91 \%$ and $77 \%$, respectively. ${ }^{41}$ However, this questionnaire demonstrated poor sensitivity and specificity in patients with $\mathrm{PD} .{ }^{41}$ Another problem common to all RBD questionnaires is that some patients are unable to complete the questionnaires, or they live alone and are unaware of their parasomnia. Finally, given the low prevalence of symptomatic RBD in the general population and assuming a questionnaire with a sensitivity and specificity of $90 \%$, only approximately $10 \%$ of patients who screen positive will actually have RBD. ${ }^{38}$ These and other data suggest the need for comprehensive objective assessments, particularly in patients with neurologic disorders.

\section{Objective Assessments}

Actigraphic measurements in patients with $A D$ reveal alterations in sleep-wake patterns with irregular sleep-wake rhythms to a complete reversal of the day/night sleep pattern. Some of these changes can be observed in the prodromal phase of AD. ${ }^{42}$ In PD, a loss of stability in the day-to-day rest-activity pattern is associated with impaired cognitive function, ${ }^{43}$ whereas reduced circadian rhythmicity may be seen in the prodromal stage. ${ }^{44}$ Finally, patients with iRBD show increased probable napping behavior, activity fragmentation, and physical inactivity during the active period. These rest-activity pattern alterations have been associated with an increased risk of phenoconversion to an overt $\alpha$ synucleinopathy. ${ }^{45}$

Video-PSG assessments in patients with $A D$ reveal reduced sleep efficiency and alterations in sleep macroarchitecture and microarchitecture (summarized in Table 1). As neurodegeneration progresses, electrographic features defining NREM stage 2 (N2) may disappear completely, rendering NREM stage 1 (N1) and N2 sleep practically indistinguishable. In this case, indeterminate NREM sleep may be scored. ${ }^{34}$ This indeterminate NREM sleep may increase further with the disappearance of the slow waves in later stages of the disease. ${ }^{46,47}$

In patients with $\mathrm{PD}$, the reduction in slow-wave sleep may be associated with increased periodic leg movements in sleep. ${ }^{48}$ In addition, lower sigma power in NREM sleep may be predictive for cognitive impairment in PD. ${ }^{50}$ In patients with $\mathrm{RBBD}$, smaller densities of fast sleep spindles and larger densities of slow spindles are described. ${ }^{51}$

The diagnosis of an RBD requires the presence of muscle activity during REM sleep (REM sleep without atonia [RSWA]). RSWA shows some degree of night-to-night stability so that a single night of PSG is considered sufficient for the diagnosis of RBD. ${ }^{38}$ Obstructive sleep apnea (OSA) is a common comorbidity in AD and PD. Importantly, patients with OSA may show motor events associated with respiratory effort in REM sleep,

\begin{tabular}{lll}
\hline $\begin{array}{l}\text { Table } 1 \\
\text { Microarchitectural and macroarchitectural changes in Alzheimer and Parkinson disease }\end{array}$ & Parkinson disease $^{48,49}$ \\
\hline Sleep efficiency & Alzheimer disease & \\
\hline Slow wave sleep & $\downarrow$ & $\downarrow$ \\
\hline REM sleep & $\begin{array}{l}\downarrow \text { episode duration, with EEG } \\
\text { slowing }\end{array}$ & $\downarrow$ \\
\hline NREM sleep & $\begin{array}{l}\text { N1 and N2 become indeterminate, } \\
\text { later SW disappear }\end{array}$ & $\begin{array}{l}\text { Lower sigma power } \\
\text { Microarchitecture }\end{array}$ \\
$\begin{array}{l}\downarrow \text { frequency and amplitude of } \\
\text { spindles and K-complexes }\end{array}$ & $\begin{array}{l}\downarrow \text { frequency and } \\
\text { amplitude of } \\
\text { spindles }\end{array}$ \\
\hline Sleep latency & $\uparrow$ & $\uparrow$ \\
\hline
\end{tabular}

Abbreviations: sigma, $(12-15 \mathrm{~Hz})$ reflects sleep spindle activity; SW, slow waves. 
which can be misinterpreted as RBD, a finding referred to as pseudo-RBD. ${ }^{52}$ If RBD is suspected in a patient with OSA, it is recommended to repeat the diagnostic workup after OSA treatment. ${ }^{38}$

When complex neuronal networks involved in the initiation and maintenance of sleep become increasingly impaired, components of different stages may occur together, leading to the socalled state dissociations. With increasing degeneration of these networks, state dissociations may increase in severity, culminating in a status dissociatus, characterized by a complete breakdown of state-determining boundaries. ${ }^{53}$ Status dissociatus has been described in patients with $\alpha$-synucleinopathies, as well as in other neurodegenerative and secondary brain diseases that predominantly affect the thalamus. ${ }^{53}$

Objective measurements using MSLT or MWT often reveal EDS in neurodegenerative disorders. Mean sleep latency was found to be significantly reduced in patients with $A D$ when compared with healthy controls and to correlate with cognitive impairment. ${ }^{54}$ Up to $50 \%$ of patients with PD suffer from EDS, ${ }^{55}$ which is often underappreciated by patients. ${ }^{37}$

\section{NEUROIMMUNOLOGICAL DISORDERS}

Many neuroimmunological disorders affect CNS areas involved in regulating sleep, wakefulness, or circadian functions. In the case of narcolepsy type 1 , a very specific group of neurons expressing hypocretin (orexin) in the hypothalamus is lost, ${ }^{56,57}$ probably secondary to autoreactive CD4+ and CD8+ T cells. ${ }^{58}$ Hypocretin neurons are essential for stabilizing the state of wakefulness, so their loss adversely affects the ability to maintain alertness. ${ }^{57}$

In contrast to a specific cell type affected in narcolepsy following a single inflammatory phase, multiple sclerosis (MS) is characterized by intermittent or chronic inflammatory-induced demyelination affecting different neuronal systems. Depending on the extent and localization of the lesions, a diverse range of symptoms can be observed, including SWCD. For example, MS lesions affecting the hypothalamus can result in secondary narcolepsy, whereas spinal lesions may trigger RLS or periodic limb movements in sleep. ${ }^{59,60}$

\section{Subjective Assessments}

The cardinal symptom in narcolepsy type 1 is cataplexy: sudden, short episodes with bilateral loss of muscle tone triggered by emotion. Although cataplexy is absent in narcolepsy type 2, all patients with narcolepsy complain of $\mathrm{EDS}^{61}$ and may report disturbed sleep, episodes of sleep paralysis, and hypnagogic (= while falling asleep) or hypnopompic (= while awakening) hallucinations. ${ }^{56}$ Specific questionnaires for narcolepsy, such as the Swiss narcolepsy scale, ${ }^{62}$ address this unique constellation of symptoms. In contrast, the Epworth sleepiness scale only assesses the symptom of EDS and shows high mean scores in patients with narcolepsy (17 \pm 3 of 24). ${ }^{61}$

Fatigue in MS is found in greater than $85 \%$ of patients $^{63}$ and can be assessed by the Fatigue Severity Scale or by the Fatigue Scale for Motor and Cognitive Functions questionnaire developed for MSrelated fatigue. ${ }^{64}$ In contrast to fatigue, EDS is less consistent at the group level in MS, although it still may be present in a substantial subpopulation. ${ }^{65}$ Pathologic fatigue and sleepiness in patients with MS was significantly associated with positive screenings for SDB, RLS, and insomnia in a large, questionnaire-based study. ${ }^{66}$ Comorbid SDB is present in $12 \%$ to $80 \%$ of patients with MS, a prevalence likely exceeding that observed in the general population. ${ }^{59,67}$ Finally, the prevalence of RLS was reported to be 4 times higher in patients with MS than in the general population. ${ }^{59,68}$

Patients with autoimmune encephalitis commonly suffer from sleep complaints such as insomnia, hypersomnolence, dream enactment behaviors, or frequent arousals. ${ }^{69}$ The anti-IgLON5 syndrome is a rare autoimmune disease characterized by sleep-wake disturbances, including insomnia, excessive sleepiness, RBD, SDB, and neurologic manifestations such as bulbar dysfunction and gait and cognitive problems. ${ }^{70}$

\section{Objective Assessments}

Sleep-wake examinations involving PSG and MSLT assessments play a critical role for the diagnostic criteria of narcolepsy according to the International Classification of Sleep Disorders, third edition. ${ }^{71}$ For example, the PSG may reveal a REM sleep latency within 15 minutes after sleep onset (sleep onset REM) in up to $50 \%$ of patients. ${ }^{56}$ Furthermore, sleep fragmentation and reduced sleep efficiency are also characteristic for narcolepsy. ${ }^{56}$ Current diagnostic criteria of narcolepsy rely on MSLT findings, which should document a mean sleep latency of 8 minutes or less and at least 2 naps with REM sleep. ${ }^{71}$

In patients with MS, PSG may reveal an increased arousal index and reduced sleep efficiency. ${ }^{72}$ The MSLT can help to further evaluate sleepiness in patients with MS, for example, to exclude narcolepsy, but robust epidemiologic data on the MSLT in patients with MS are lacking. ${ }^{59}$ In autoimmune encephalitis, PSG may show sleep fragmentation, reduced sleep 
efficiency, and reduced or absent NREM stage 3 (N3) and REM sleep. ${ }^{69}$ In a small study, spindle density was shown to be decreased. ${ }^{73}$ Larger systematic studies are needed to assess associations between antibody subtypes and specific sleep disorders and the influence of sleep disorders on the clinical presentation and long-term outcome of patients with autoimmune encephalitis. In the anti-IgLON5 syndrome, the PSG may show an abnormal (undifferentiated) NREM sleep initiation, with sleep-related vocalizations and movements, followed by periods of normal NREM sleep, RBD, and OSA with stridor. ${ }^{74}$

\section{EPILEPSY}

Epilepsy is linked in a bidirectional manner to sleep. On the one hand, seizures, antiepileptic drugs, and interictal activity may alter sleep macroarchitecture and microarchitecture. On the other hand, sleep deprivation and comorbid sleep disorders may reduce the seizure threshold and limit its control. ${ }^{75}$ Moreover, up to one-half of epileptic patients report sleep complaints. ${ }^{76,77}$ Specifically, $52 \%$ complain of sleep maintenance insomnia (vs $38 \%$ in controls), whereas loud snoring and restless legs symptoms were found to be independent predictors of EDS in patients with epilepsy. ${ }^{76}$ Moderate to severe SDB affects up to $26.5 \%$ of epileptic patients and may increase seizure frequency. ${ }^{78}$ These findings highlight the importance of SWCD diagnosis and treatment in epilepsy management. In addition, several epilepsy syndromes show seizure activity exclusively or predominantly in sleep. These are termed sleep-related epilepsy.

\section{Subjective Measures}

A careful clinical history with both patient and witness is critical to correctly diagnose and establish the semiology of the ictal events. The most frequent complaints are EDS and insomnia. ${ }^{76,79}$ Specific questionnaires, such as the Frontal Lobe Epilepsy and Parasomnias scale, can help differentiate some forms of epilepsy from disorders of arousal (confusional arousal, sleepwalking and sleep terrors), although the semiological similarities pose diagnostic challenges.

\section{Objective Measures}

In case of suspicion of sleep-related epilepsy, objective measurements, such as home video recording and a video-PSG with extended EEG (10/20) montage is recommended. ${ }^{80,81}$ PSG recordings in patients with epilepsy may show sleep architectural abnormalities, such as increased number of arousals and increased wake after sleep onset, increased stage shifts, and reduced percentage of REM sleep. ${ }^{75}$ Epileptic activity is also specifically affected by sleep stage: interictal and ictal activity are increased predominantly in NREM sleep when compared with REM sleep. ${ }^{82,83}$ This association is proposed to be associated with NREM sleep characteristics, that is, increased EEG synchronization that may favor seizure propagation and muscle tone preservation during NREM sleep that allows seizure-related movements to occur. ${ }^{84}$

Although sleepiness is a frequent complaint in patients with epilepsy, objective assessments of vigilance and wakefulness are sparse, showing little correlation between subjective and objective sleepiness. ${ }^{85}$

\section{NEW FRONTIERS FOR MEASURING SLEEP- WAKE AND CIRCADIAN DISORDERS IN NEUROLOGIC DISORDERS}

Given the high prevalence of sleep disorders comorbid with neurologic disorders and their impact on the course or presentation of neurologic disease, it is essential to identify and treat SWCD to optimize neurologic management. Future advancements are needed with respect to screening, including questionnaires specifically designed for patients with neurologic disorders. Moreover, future technologies on the horizon may promote new diagnostic and management tools.

\section{Future Role of Questionnaires}

Screening tools for individual SWCD disorders are available, including the Epworth Sleepiness Scale ${ }^{86}$ to test the propensity to fall asleep, the Fatigue Severity Scale, ${ }^{87}$ Insomnia Severity Index, ${ }^{88}$ the Single Question for RLS, ${ }^{89}$ STOP-BANG score for sleep apnea, ${ }^{90}$ and the Swiss Narcolepsy Scale ${ }^{62}$ to name a few. However, a fully validated general screening instrument for patients with neurologic disorders is currently missing, making it difficult for neurologists outside of sleep medicine to select the right screening tool. Therefore, a brief domain-based questionnaire might be the future such as a first promising attempt in the SDS-25. ${ }^{91}$ The validation of a single, brief questionnaire covering a targeted spectrum of SWCD designed for patients with neurologic disorders remains an area of interest for future research.

\section{Future Frontiers in Sleep-Wake and Circadian Disorders Monitoring}

In-laboratory video PSG (ie, a level 1 sleep study) plays a central role in objective clinical sleep 


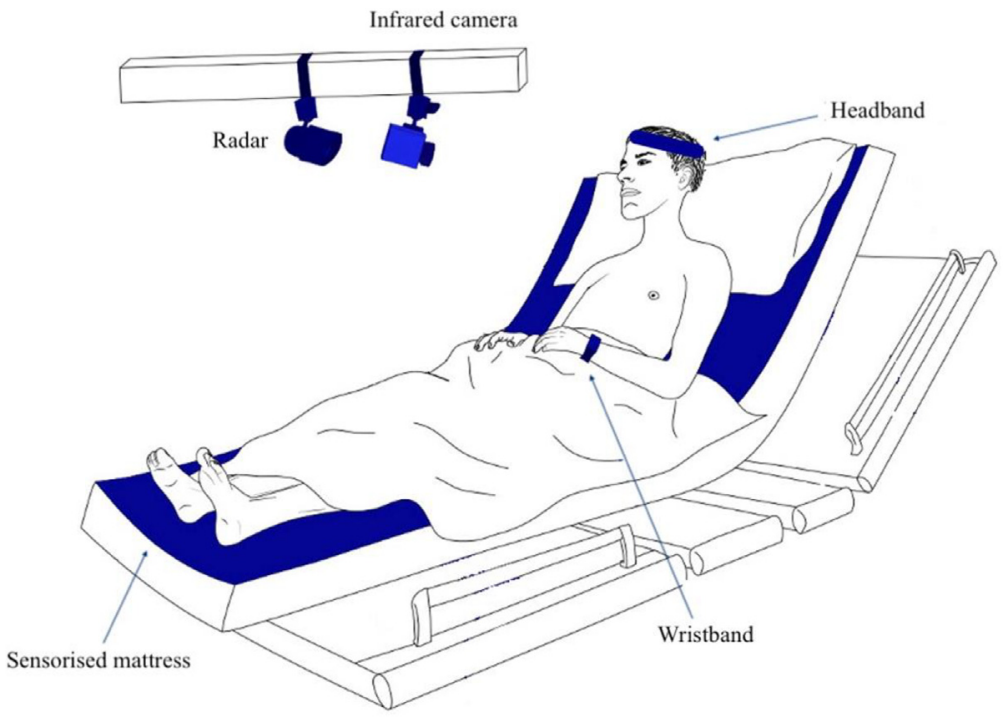

Fig. 2. Example of an unobtrusive sensor network. On top, 2 contactless technologies, radar and infrared camera. The subject lies on a sensorized mattress wearing a smart wristband and an electroencephalographicbased headband. ${ }^{93}$ assessment. Although it is the current gold standard for diagnosis of many SWCD, the unnatural environment and highly obtrusive character of the currently used devices may negatively affect sleep. In addition, this single night snapshot cannot detect periodicities or subtle changes over time as may be anticipated for many chronic or progressive neurologic disorders.

With advances in technology, less obtrusive devices address these issues and allow long-term home monitoring of sleep, wake, and circadian functions. Actigraphy was one of the first wearable devices that could measure several parameters of the sleep-wake rhythm. Novel wrist bands have included additional sensors for measuring heart rate, skin temperature, and electrodermal activity. ${ }^{92}$ To record brain activity and derive sleep stages, headbands with dry EEG electrodes have gained popularity over the last years. ${ }^{93}$ Even less invasive are contactless technologies to monitor sleep. Here we find sensorized mattresses based on ballistocardiography, which are able to extract body movements, heart rate, and respiratory rate. $^{94}$ In addition, bedside radar technology has recently been suggested as a potential future tool for sleep-wake discrimination, ${ }^{95}$ sleep stage scoring, ${ }^{96}$ and detection of SDB. ${ }^{97}$ Although skin temperature changes occur across sleep and wake states, the potential roles of other technologies such as thermal cameras or electrodermal activity remain unclear.

Several studies have shown a high correlation between such wearable and nearable devices and PSG. ${ }^{98}$ However, studies mostly compared one single device to PSG in healthy participants. As a result, there is lack of ground truth data on
2 levels: first, each device records specific types of data, but it remains unclear what combination of sensing devices may be optimal. Second, studies are lacking to validate such devices as shown in Fig. 2 in both medical and home settings with patients with sleep or neurologic disorders. The medical application of currently available commercial smartwatches has been complicated by the lack of harmonization of technologies across devices. However, if these and other wristband devices become validated in the future, the medical setting for clinic investigation will likely shift from an in-laboratory setting to the home environment. Theoretically, a combined sensor network offers the potential for long-term, inhome monitoring with a similar diagnostic efficacy as PSG. At our institution a new unit called NeuroTec was recently inaugurated to test and validate new approaches for wearable and nearable approaches to long-term and home monitoring of SWCD and other disturbances in neurologic patients. $^{99}$

\section{SUMMARY}

Neurologic disorders often affect sleep-wake and circadian patterns, either as a primary consequence of brain lesions that disrupt neuronal networks regulating sleep or circadian time or as a secondary consequence of underlying sensory or motor neuropathology. These SWCD may present in diverse ways, ranging from changes to sleep structure or EEG microarchitecture without subjective symptoms up to the complete dissociation of the sleep-wake stages in advanced neurodegeneration. Identifying these symptoms using 
both subjective and objective assessments allows inference into the site of the lesion and progression of disease. Importantly, diagnosis and treatment of SWCD can optimize management of neurologic diseases.

The impact of neurologic disorders on SWCD is diverse as can be measured using various methods. Available tools not only provide objective assessments of nighttime sleep but also can provide a long-term view of circadian activity patterns through actigraphy or wearable devices. Moreover, objective daytime testing can measure diurnal symptoms that may result from SWCD. Finally, new technologies may potentially facilitate future long-term monitoring of chronic neurologic patients while retaining a high resolution of recording fidelity, thereby allowing optimization of clinical management and monitoring of treatment efficacy.

\section{DISCLOSURE}

S. Baillieul received support from the European Respiratory Society Fellowship through a LongTerm Research Fellowship (Fellowship ID number: LTRF202001-00711). C.L.A. Bassetti received support from the Swiss National Science Foundation grant titled Early Sleep Apnea Treatment in Stroke: A Randomized, Rater-Blinded, Clinical Trial of Adaptive Servo-Ventilation (Grant ID number: 33IC30_166827). M.H. Schmidt and C.L.A. Bassetti received support from the Interfaculty Research Cooperation (IRC): Decoding Sleep, University of Bern, Bern, Switzerland (Grant Identifier: 41-050). M.H. Schmidt received support from Innosuisse (Grant Identifier: 30664.1 IP-LS).

\section{REFERENCES}

1. Feigin VL, Vos T, Nichols E, et al. The global burden of neurological disorders: translating evidence into policy. Lancet Neurol 2020;19(3):255-65.

2. Gottlieb E, Landau E, Baxter H, et al. The bidirectional impact of sleep and circadian rhythm dysfunction in human ischaemic stroke: a systematic review. Sleep Med Rev 2019;45:54-69.

3. Hermann DM, Bassetti CL. Sleep-related breathing and sleep-wake disturbances in ischemic stroke. Neurology 2009;73(16):1313-22.

4. Bassetti CLA, Randerath W, Vignatelli L, et al. EAN/ ERS/ESO/ESRS statement on the impact of sleep disorders on risk and outcome of stroke. Eur J Neurol 2020;27(7):1117-36.

5. Duss SB, Seiler A, Schmidt MH, et al. The role of sleep in recovery following ischemic stroke: a review of human and animal data. Neurobiol Sleep Circadian Rhythms 2017:2:94-105.
6. Hermann DM, Siccoli M, Brugger P, et al. Evolution of neurological, neuropsychological and sleepwake disturbances after paramedian thalamic stroke. Stroke 2008;39(1):62-8.

7. Vock J, Achermann P, Bischof M, et al. Evolution of sleep and sleep EEG after hemispheric stroke. J Sleep Res 2002;11(4):331-8.

8. Dekkers M, Alexiev F, Denier N, et al. Sleep duration increases after stroke: a prospective study of 438 patients. Eur J Neurol 2020;27(suppl. 1):252.

9. Wu W, Cui L, Fu Y, et al. Sleep and cognitive abnormalities in acute minor thalamic infarction. Neurosci Bull 2016;32(4):341-8.

10. Maestri M, Romigi A, Schirru A, et al. Excessive daytime sleepiness and fatigue in neurological disorders. Sleep Breath 2020;24(2):413-24.

11. Bassetti CL. Sleep and stroke. In: Sleep medicine Textbook. 2nd Edition. European Sleep Research Society (ESRS); 2021. p. 959-70.

12. Hasan F, Gordon C, Wu D, et al. Dynamic prevalence of sleep disorders following stroke or transient ischemic Attack: systematic review and meta-analysis. Stroke 2021;52(2):655-63.

13. Chen X, Bi H, Zhang M, et al. Research of sleep disorders in patients with acute cerebral infarction. J Stroke Cerebrovasc Dis 2015;24(11):2508-13.

14. Boulos MI, Murray BJ, Muir RT, et al. Periodic limb movements and white matter Hyperintensities in first-Ever minor stroke or high-risk transient ischemic Attack. Sleep 2017;40(3). https://doi.org/10.1093/ sleep/zsw080.

15. Takala M, Puustinen J, Rauhala E, et al. Pre-screening of sleep-disordered breathing after stroke: a systematic review. Brain Behav 2018;8(12):e01146.

16. Brown DL, He K, Kim S, et al. Prediction of sleepdisordered breathing after stroke. Sleep Med 2020; 75:1-6.

17. Takekawa H, Miyamoto M, Miyamoto T, et al. Circadian rhythm abnormalities in the acute phase of cerebral infarction correlate with poor prognosis in the chronic phase. Auton Neurosci 2007;131(1-2):131-6.

18. Kwon S, Kim H, Yeo W-H. Recent advances in wearable sensors and portable electronics for sleep monitoring. iScience 2021;24(5):102461.

19. Bassetti CL, Aldrich MS. Sleep electroencephalogram changes in acute hemispheric stroke. Sleep Med 2001;2(3):185-94.

20. Pace M, Camilo MR, Seiler A, et al. Rapid eye movements sleep as a predictor of functional outcome after stroke: a translational study. Sleep 2018;41(10). https://doi.org/10.1093/sleep/zsy138.

21. Bassetti CL, Hermann DM. Sleep and stroke. Handbook Clin Neurol 2011;99:1051-72. https://doi.org/ 10.1016/B978-0-444-52007-4.00021-7. Elsevier.

22. Bassetti C, Mathis J, Gugger M, et al. Hypersomnia following paramedian thalamic stroke: a report of 12 patients. Ann Neurol 1996;39(4):471-80. 
23. Mensen A, Poryazova R, Huber R, et al. Individual spindle detection and analysis in high-density recordings across the night and in thalamic stroke. Sci Rep 2018;8(1):17885.

24. Gottselig JM, Bassetti CL, Achermann P. Power and coherence of sleep spindle frequency activity following hemispheric stroke. Brain 2002;125(2): 373-83.

25. Poryazova R, Huber R, Khatami R, et al. Topographic sleep EEG changes in the acute and chronic stage of hemispheric stroke. J Sleep Res 2015;24(1):54-65.

26. Jaramillo V, Jendoubi J, Maric A, et al. Thalamic influence on slow wave slope renormalization during sleep. Ann Neurol 2021. https://doi.org/10.1002/ ana.26217.

27. Diekelmann S, Born J. The memory function of sleep. Nat Rev Neurosci 2010;11(2):114-26. https://doi.org/10.1038/nrn2762.

28. Tononi G, Cirelli C. Sleep and the price of plasticity: from synaptic and cellular homeostasis to memory consolidation and integration. Neuron 2014;81(1): 12-34.

29. Facchin L, Schöne C, Mensen A, et al. Slow waves promote sleep-dependent plasticity and functional recovery after stroke. J Neurosci 2020;40(45): $8637-51$.

30. Ding Q, Whittemore R, Redeker N. Excessive daytime sleepiness in stroke Survivors: an integrative review. Biol Res Nurs 2016;18(4):420-31.

31. Kim H, Young T. Subjective daytime sleepiness: Dimensions and correlates in the general population. Sleep 2005;28(5):625-34.

32. Peter-Derex L, Yammine P, Bastuji H, et al. Sleep and Alzheimer's disease. Sleep Med Rev 2015;19:29-38.

33. Landolt H-P, Glatzel M, Blattler T, et al. Sleep-wake disturbances in sporadic Creutzfeldt-Jakob disease. Neurology 2006;66(9):1418-24.

34. Santamaria J, Hogl B, Trenkwalder C, et al. Scoring sleep in neurological patients: the need for specific considerations. Sleep 2011;34(10):1283-4.

35. Sani TP, Bond RL, Marshall CR, et al. Sleep symptoms in syndromes of frontotemporal dementia and Alzheimer's disease: a proof-of-principle behavioural study. eNeurologicalSci 2019;17:100212. https://doi.org/10.1016/j.ensci.2019.100212.

36. Rothman SM, Mattson MP. Sleep disturbances in Alzheimer's and Parkinson's diseases. Neuromol Med 2012;14(3):194-204.

37. Bargiotas $\mathrm{P}$, Lachenmayer ML, Schreier DR, et al. Sleepiness and sleepiness perception in patients with Parkinson's disease: a clinical and electrophysiological study. Sleep 2019;42(4). https://doi.org/10. 1093/sleep/zsz004.

38. Dauvilliers Y, Schenck CH, Postuma RB, et al. REM sleep behaviour disorder. Nat Rev Dis Primers 2018;4(1):19.
39. Galbiati A, Carli G, Hensley M, et al. REM sleep behavior disorder and Alzheimer's disease: Definitely No relationship? J Alzheimers Dis 2018;63(1):1-11.

40. Yang $X$, Liu B, Shen $H$, et al. Prevalence of restless legs syndrome in Parkinson's disease: a systematic review and meta-analysis of observational studies. Sleep Med 2018;43:40-6.

41. Li K, Li S-H, Su W, et al. Diagnostic accuracy of REM sleep behaviour disorder screening questionnaire: a meta-analysis. Neurol Sci 2017;38(6):1039-46.

42. Musiek ES, Bhimasani M, Zangrilli MA, et al. Circadian rest-activity pattern changes in aging and Preclinical Alzheimer disease. JAMA Neurol 2018;75(5):582.

43. Wu JQ, Li P, Stavitsky Gilbert K, et al. Circadian restactivity rhythms Predict cognitive function in early Parkinson's disease independently of sleep. Mov Disord Clin Pract 2018;5(6):614-9.

44. Leng Y, Blackwell T, Cawthon PM, et al. Association of circadian abnormalities in Older Adults with an increased risk of developing Parkinson disease. JAMA Neurol 2020;77(10):1270.

45. Feng $\mathrm{H}$, Chen L, Liu Y, et al. Rest-activity pattern alterations in idiopathic REM sleep behavior disorder. Ann Neurol 2020;88(4):817-29.

46. Petit D, Gagnon J-F, Fantini ML, et al. Sleep and quantitative EEG in neurodegenerative disorders. J Psychosom Res 2004;56(5):487-96.

47. McCurry SM, Ancoli-Israel S. Sleep dysfunction in Alzheimer's disease and other dementias. Curr Treat Options Neurol 2003;5(3):261-72.

48. Zhang F, Niu L, Liu X, et al. Rapid eye movement sleep behavior disorder and neurodegenerative diseases: an Update. Aging Dis 2020;11(2):315.

49. Christensen JAE, Nikolic M, Warby SC, et al. Sleep spindle alterations in patients with Parkinson's disease. Front Hum Neurosci 2015;9. https://doi.org/ 10.3389/fnhum.2015.00233.

50. Latreille V, Carrier J, Gaudet-Fex B, et al. Electroencephalographic prodromal markers of dementia across conscious states in Parkinson's disease. Brain 2016;139(4):1189-99.

51. O'Reilly C, Godin I, Montplaisir J, et al. REM sleep behaviour disorder is associated with lower fast and higher slow sleep spindle densities. J Sleep Res 2015;24(6):593-601.

52. Iranzo A, Santamaría J. Severe obstructive sleep apnea/Hypopnea Mimicking REM sleep behavior disorder. Sleep 2005;28(2):203-6.

53. Antelmi E, Ferri R, Iranzo A, et al. From state dissociation to status dissociatus. Sleep Med Rev 2016; 28:5-17.

54. Bonanni E, Maestri M, Tognoni G, et al. Daytime sleepiness in mild and moderate Alzheimer's disease and its relationship with cognitive impairment. J Sleep Res 2005;14(3):311-7.

55. Arnulf I. Excessive daytime sleepiness in parkinsonism. Sleep Med Rev 2005;9(3):185-200. 
56. Bassetti CLA, Adamantidis A, Burdakov D, et al. Narcolepsy - clinical spectrum, aetiopathophysiology, diagnosis and treatment. Nat Rev Neurol 2019; 15(9):519-39.

57. Adamantidis AR, Schmidt MH, Carter ME, et al. A circuit perspective on narcolepsy. Sleep 2020; 43(5):zsz296.

58. Latorre D, Kallweit U, Armentani E, et al. T cells in patients with narcolepsy target self-antigens of hypocretin neurons. Nature 2018;562(7725):63-8.

59. Veauthier C. Sleep disorders in multiple sclerosis. Review. Curr Neurol Neurosci Rep 2015;15(5):21.

60. Kallweit U, Bassetti CLA, Oberholzer M, et al. Coexisting narcolepsy (with and without cataplexy) and multiple sclerosis: Six new cases and a literature review. J Neurol 2018;265(9):2071-8.

61. Luca G, Haba-Rubio J, Dauvilliers Y, et al. Clinical, polysomnographic and genome-wide association analyses of narcolepsy with cataplexy: a European Narcolepsy Network study. J Sleep Res 2013; 22(5):482-95.

62. Bargiotas P, Dietmann A, Haynes AG, et al. The Swiss Narcolepsy Scale (SNS) and its short form (sSNS) for the discrimination of narcolepsy in patients with hypersomnolence: a cohort study based on the Bern Sleep-Wake Database. J Neurol 2019; 266(9):2137-43.

63. Krupp LB, Alvarez LA, LaRocca NG, et al. Fatigue in multiple sclerosis. Arch Neurol 1988;45(4):435-7.

64. Penner I, Raselli C, Stöcklin M, et al. The Fatigue Scale for Motor and Cognitive Functions (FSMC): validation of a new instrument to assess multiple sclerosis-related fatigue. Mult Scler 2009;15(12): 1509-17.

65. Popp RFJ, Fierlbeck AK, Knüttel H, et al. Daytime sleepiness versus fatigue in patients with multiple sclerosis: a systematic review on the Epworth sleepiness scale as an assessment tool. Sleep Med Rev 2017;32:95-108.

66. Brass SD, Li C-S, Auerbach S. The Underdiagnosis of sleep disorders in patients with multiple sclerosis. J Clin Sleep Med 2014;10(09): 1025-31.

67. Caminero A, Bartolomé M. Sleep disturbances in multiple sclerosis. J Neurol Sci 2011;309(1-2):86-91.

68. Italian REMS Study Group, Manconi M, FeriniStrambi L, Filippi M, et al. Multicenter case-control study on restless legs syndrome in multiple sclerosis: the REMS study. Sleep 2008;31(7):944-52.

69. Blattner MS, Day GS. Sleep disturbances in patients with autoimmune encephalitis. Curr Neurol Neurosci Rep 2020;20(7):28.

70. Gaig C, Graus F, Compta Y, et al. Clinical manifestations of the anti-IgLON5 disease. Neurology 2017; 88(18):1736-43.

71. American Academy of Sleep Medicine, editor. International Classification of sleep disorders. 3. ed. American Acad. of Sleep Medicine; 2014.
72. Tanioka K, Castelnovo A, Tachibana N, et al. Framing multiple sclerosis under a polysomnographic perspective. Sleep. Published online October 22, 2019:zsz232. doi:10.1093/sleep/zsz232

73. Serdaroglu E, Tezer FI, Saygi S. Autoimmune epilepsy and/or limbic encephalitis can lead to changes in sleep spindles. Noro Psikiyatr Ars 2017;8. https:// doi.org/10.5152/npa.2017.19442. Published online September.

74. Gaig C, Iranzo A, Santamaria J, et al. The sleep disorder in anti-lgLON5 disease. Curr Neurol Neurosci Rep 2018;18(7):41.

75. Kataria L, Vaughn BV. Sleep and epilepsy. Sleep Med Clin 2016;11(1):25-38.

76. Khatami R, Zutter D, Siegel A, et al. Sleep-wake habits and disorders in a series of 100 adult epilepsy patients - a prospective study. Seizure 2006; 15(5):299-306.

77. Matsuoka E, Saji M, Kanemoto K. Daytime sleepiness in epilepsy patients with special attention to traffic accidents. Seizure 2019;69:279-82.

78. Sivathamboo S, Perucca P, Velakoulis D, et al. Sleep-disordered breathing in epilepsy: epidemiology, mechanisms, and treatment. Sleep 2018; 41(4). https://doi.org/10.1093/sleep/zsy015.

79. Quigg M, Gharai S, Ruland J, et al. Insomnia in epilepsy is associated with continuing seizures and worse quality of life. Epilepsy Res 2016;122:91-6. https://doi.org/10.1016/j.eplepsyres.2016.02.014.

80. Nobili L, Weerd A, Rubboli G, et al. Standard procedures for the diagnostic pathway of sleep-related epilepsies and comorbid sleep disorders: an EAN, ESRS and ILAE-Europe consensus review. Eur J Neurol 2021;28(1):15-32.

81. Wu T, Avidan AY, Engel J. Sleep and epilepsy, clinical spectrum and Updated review. Sleep Med Clin 2021;16(2):389-408.

82. Frauscher B, von Ellenrieder N, Ferrari-Marinho T, et al. Facilitation of epileptic activity during sleep is mediated by high amplitude slow waves. Brain 2015;138(Pt 6):1629-41.

83. Zubler F, Rubino A, Lo Russo G, et al. Correlating interictal Spikes with sigma and Delta Dynamics during non-rapid-eye-movement-sleep. Front Neurol 2017;8:288. https://doi.org/10.3389/fneur.2017. 00288.

84. Shouse MN, Farber PR, Staba RJ. Physiological basis: how NREM sleep components can promote and REM sleep components can suppress seizure discharge propagation. Clin Neurophysiol 2000;111:S9-18. https://doi.org/10.1016/S1388-2457(00)00397-7.

85. Drake ME, Weate SJ, Newell SA, et al. Multiple sleep latency tests in epilepsy. Clin Electroencephalogr 1994;25(2):59-62.

86. Johns MW. A new method for measuring daytime sleepiness: the Epworth sleepiness scale. Sleep 1991;14(6):540-5. 
87. Krupp LB. The fatigue severity scale: application to patients with multiple sclerosis and systemic Lupus Erythematosus. Arch Neurol 1989;46(10):1121.

88. Gagnon C, Belanger L, Ivers $H$, et al. Validation of the insomnia severity index in primary care. J Am Board Fam Med 2013;26(6):701-10.

89. Ferri R, Lanuzza B, Cosentino Fll, et al. A single question for the rapid screening of restless legs syndrome in the neurological clinical practice. Eur J Neurol 2007;14(9):1016-21.

90. Chung F, Subramanyam R, Liao P, et al. High STOPBang score indicates a high probability of obstructive sleep apnoea. Br J Anaesth 2012;108(5): 768-75.

91. Klingman JK, Jungquist CR, Perlis ML. Introducing the sleep disorders symptom Checklist-25: a primary care Friendly and comprehensive screener for sleep disorders. Sleep Med Res 2017;8(1): 17-25.

92. De Zambotti M, Cellini N, Goldstone A, et al. Wearable sleep technology in clinical and research settings. Med Sci Sports Exerc 2019;51(7):1538-57.

93. Arnal PJ, Thorey V, Debellemaniere E, et al. Original Article the Dreem Headband compared to polysomnography for electroencephalographic signal acquisition and sleep staging. Sleep 2020;1-13. https://doi.org/10.1093/sleep/zsaa097.

94. Xie Q, Wang M, Zhao Y, et al. A Personalized Beatto-Beat heart rate detection system from Ballistocardiogram for smart home applications. IEEE Trans Biomed Circuits Syst 2019;13(6):1593-602.

95. Heglum HSA, Kallestad $H$, Vethe D, et al. Distinguishing sleep from wake with a radar sensor: a contactfree real-time sleep monitor. Sleep 2021;44(8). https://doi.org/10.1093/sleep/zsab060. zsab060.

96. Toften S, Pallesen S, Hrozanova M, et al. Validation of sleep stage classification using non-contact radar technology and machine learning (Somnofy ${ }^{\circledR}$ ). Sleep Med 2020;75:54-61. https://doi.org/10.1016/ j.sleep.2020.02.022.

97. Zhou Y, Shu D, Xu H, et al. Validation of novel automatic ultra-wideband radar for sleep apnea detection. J Thorac Dis 2020;12(4):1286-95.

98. Mantua J, Gravel N, Spencer RMC. Reliability of sleep measures from four personal health monitoring devices compared to research-based actigraphy and polysomnography. Sensors (Switzerland) 2016;16(5). https://doi.org/10.3390/s16050646.

99. Schindler KA, Nef T, Baud MO, et al. NeuroTec Sitem-Insel bern: Closing the last mile in Neurology. CTN 2021;5(2):13. 